\title{
Effectiveness of binocular therapy as a complementary treatment of part-time patching in older amblyopic children: A randomized clinical trial
}

\section{Qing Zhu ( $\nabla$ zhuqingzqzq@sina.com )}

The Second Hospital of Dalian Medical University https://orcid.org/0000-0002-5950-2465

\section{Qi Zhao}

The Second Hospital of Dalian Medical University https://orcid.org/0000-0001-7945-6649

\section{Ran Liang}

The Second Hospital of Dalian Medical University

\section{Xin $\mathrm{He}$}

The Second Hospital of Dalian Medical University

\section{Ming-Jun Gao}

The Second Hospital of Dalian Medical University

\section{Research Article}

Keywords: amblyopia, residual amblyopia, binocular vision, stereo acuity, visual development

Posted Date: March 7th, 2022

DOI: https://doi.org/10.21203/rs.3.rs-1181232/v1

License: (9) This work is licensed under a Creative Commons Attribution 4.0 International License. Read Full License 


\section{Abstract}

PURPOSE To assess the effectiveness of combined use of stereoscopic 3D video movies and part-time patching in treating older amblyopic children with poor response or compliance to traditional patching treatments and comparing this combined treatment with patching alone.

Methods Thirty-two children aged 5-12 years with amblyopia associated with anisometropia, strabismus, or both were recruited in a randomized clinical trial. Eligible participants were assigned randomly to the combined and patching groups. Here, binocular treatment refers to using the Bangerter filter to blur the fellow eye and subsequently watching a close-up 3D movie with large parallax. The primary outcome was amblyopic-eye (AE) best-corrected visual acuity (BCVA) improvement at six weeks. In addition, secondary outcomes included BCVA of AE improvement at three weeks and change of stereo acuity.

Results Of 32 participants, mean (SD) age was 6.63 (1.46) years, and 19 (59\%) were female. At 6-weeks,

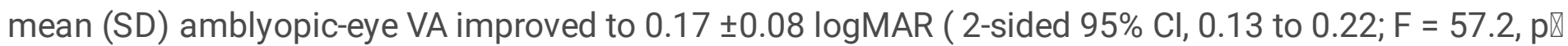
0.01 ) and $0.05 \pm 0.04 \log$ MAR (2-sided $95 \% \mathrm{Cl}, 0.05$ to $0.09 ; \mathrm{F}=8.73, \mathrm{p}=0.01$ ) in the combined and patching groups, respectively. The difference was statistically significant (mean difference, 0.13 logMAR [1.3 line]; $95 \% \mathrm{Cl}, 0.08-0.17$ logMAR [0.8-1.7 lines]; t25 = 5.65, $\mathrm{p}<0.01$ ). After treatment, only the combined group had significantly improved stereo acuity, such as binocular function score (median [interquartile range], 2.30 [2.23 to 2.68] vs. 1.69 [1.60 to 2.30] log arcsec; paired, $z=-3.53, p \otimes 0.01$ ), and mean stereo acuity gain was $0.47 \mathrm{log}$ arcsec $( \pm 0.22)$. Changes in other types of stereo acuity were similar.

Conclusion Our laboratory-based binocular treatment strategy engaged a high level of compliance that led to a substantial gain in visual function after a short period of treatment for older amblyopic children having poor response or compliance to traditional patching treatments. Notably, the improving stereo acuity showed a greater advantage.

\section{Introduction}

Amblyopia is a visual developmental disorder that arises from abnormal visual experience during the first years of life. Amblyopia can be bilateral but most commonly considered a monocular disease that affects the vision of one eye of children with strabismus, anisometropia, or both. The mechanism involved is deprivation-induced response depression and deprivation-enabled, experience-dependent response potentiation. [1, 2] However, recent studies have found that in addition to decreased visual acuity, amblyopia is also accompanied by other vision dysfunctions such as impaired stereo acuity, deficits of spatial vision and motion perception.[3-5]

For hundreds of years, treatment for amblyopia relied on depriving the fellow eye of vision and forcing the use of the amblyopic eye (e.g., patching, [6, 7] atropine drops, [8] or Bangerter filters [9]) to promote development or recovery of visual acuity. Although $73-90 \%$ of children with amblyopia show improved visual acuity with various treatment modalities alone or combinations, $15-50 \%$ failed to achieve normal 
visual acuity after extended treatment periods. [6, 9-12] Moreover, patients who achieved normal visual acuity with traditional treatment, the binocular dysfunctions [3,13], and risk of amblyopia recurrence after cessation of treatment still existed. $[14,15]$ While amblyopia is traditionally diagnosed and treated as a monocular condition, researchers now consider amblyopia related to abnormal binocular visual system development early in life. $[16,17]$ This may explain why the conventional patching treatment improves amblyopic eye visual acuity more than binocular vision, and residual and recurrent amblyopia may persist even after monocular treatment.

In experimental strabismus and anisometropia amblyopic macaques, cortical binocularity was reduced. $[18,19]$ Most of the cells innervated by the binocular were in an inactive state, but the binocular cortical communication in patients with amblyopia persisted.[20,21] Therefore, researchers worked to develop novel approaches for amblyopia treatment. Binocular treatment encourages binocular summation and might involve neural plasticity in brain regions involved in binocular vision, such as the parietal cortex. Binocular treatment of amblyopia involves any treatment where both eyes are being used, and the amblyopic eye is the primary eye performing a given visual task. Investigations showed that perceptual learning could improve visual acuity, stereopsis, and other visual functions in adults and older children with amblyopia.[22-24] However, patients need to perform thousands of deep sine wave gratings or other simple stimulus practice tests. Dichoptic therapies, first proposed by Hess et al [25], uses dichoptic contrast balance, where the contrast level of the dominant eye is reduced to negate suppression to a level where the contrast sensitivity of the two eyes is equal and balanced in the pursuit of the given visual task.

Although these binocular treatments are effective, none showed outstanding advantages compared with traditional patching treatments.

$[26,27]$ Some characteristics in these studies include: (1) Lack of compliance in the large-scale research (simple games were too boring, while complex games were not easy to participate even for older children); (2) Younger children have better therapeutic effects than older children, and the therapeutic effects for adults with amblyopia are limited; (3) Patients with a history of patching treatment are poorly treated. There is sufficient evidence to develop more engaging binocular treatments as adjuvant treatments for older children with failed traditional treatment, to improve their vision and binocular visual function. Brief exposures to a three-dimensional (3D) stereoscopic environment with greater disparities than encountered in natural scenes can restore stereopsis.[28] Further research showed that playing immersive 3D video games might have potential therapeutic value in the recovery of reduced stereopsis in amblyopia.[29] Our study used a Bangerter filter to remove this obstacle to binocular vision and then provided eye-catching visual stimulation (movie videos with better patient compliance) to immerse patients in a 3D environment with enormous disparities. The effects of 3D video training on visual functions, especially stereo acuity, were investigated in older children with residual amblyopia.

\section{Materials And Methods}




\subsection{Participants}

Children aged 5-12 years with amblyopia associated with anisometropia, strabismus, or both were recruited from the Pediatric Ophthalmology Clinic, the Second Affiliated Hospital of Dalian Medical University. Our research followed the tenets of the Declaration of Helsinki. Informed consent from caregivers and assent from children was obtained before enrollment and approved by the Ethics Committee of the Second Affiliated Hospital of Dalian Medical University.

The diagnosis was according to amblyopia preferred practice pattern.[30] Amblyopia, defined as the bestcorrected visual acuity (BCVA) of the amblyopic eye, was worse than $0.2 \log M A R$ and fellow eye BCVA equal or better than $0.1 \log M A R$, and an interocular acuity difference of $0.2 \log M A R$ or greater.

Inclusion criteria included: (1) 5-12 years of age; (2) amblyopia associated with anisometropia, strabismus, or both; (3) anisometropia was defined as $\geq 0.50 \mathrm{D}$ difference between eyes in spherical power or $\geq 1.00 \mathrm{D}$ difference between eyes in cylindrical power in any meridian; (4) children with strabismus were initially diagnosed with esotropia but were aligned with surgery or spectacle correction to no more than 10 prism diopters using a prism and alternate cover test (PACT) at near; (5) persistent minimum of 16 weeks of refractive error correction; (6) the patient received patching treatment in the past and had a residual visual acuity deficit, with the vision not improving for more than six months (defined as $<0.1 \log$ MAR change by the same testing method measured on two consecutive reviews $\geq 3$ months apart).[31]

Exclusion criteria included: (1) any pathological conditions that may reduce visual acuity determined by ocular examination performed within the past seven months; (2) previous intraocular or refractive surgery; (3) significant neurological or behavioral problems; (4) severe developmental delay that would interfere with treatment or evaluation (in the opinion of the investigator).

\subsection{Randomization}

Randomization was performed by a researcher masked who provided individual sealed sequentially numbered envelopes. Randomization schedules were created using a random number generator function and randomly assigned participants according to a ratio of 2:3. After confirming eligibility and obtaining written informed consent, researchers opened the sealed envelope, enrolled the child, and set them to the appropriate treatment.

\subsection{Treatment Protocols}

\subsubsection{Study Visits and Testing Procedures}

Treatment groups followed the same treatment schedule and vision assessment. During the baseline visit, admission qualifications were determined, and vision assessments were conducted. Children were 
assigned randomly to patching and binocular treatment (Combined group) or patching treatment alone (Patching group) for six weeks. Follow-up visits were planned at 3 and 6 weeks after the baseline visit. At each visit, BCVA was measured on each eye with optimal refractive correction (if applicable) and without cycloplegia by a study-certified examiner using the Tumbling E Early Treatment Diabetic Retinopathy Study chart (Precision Vision, Inc, USA). Stereo acuity was measured using Randot Preschool Stereoacuity Test (Stereo Optical Company, USA) and Fly Stereo Acuity Test with LEA Symbols (Vision Assessment Corporation, USA) at $40 \mathrm{~cm}$. Distance Stereo acuity was measured using the method of evaluating stereo acuity with 3D shutter glasses technology[32] at $3.1 \mathrm{~m}$. Binocular sensory status was measured using The Worth 4 dot test. All measurements were conducted by study-certified examiners masked.

\subsubsection{Binocular treatment protocol}

In the experiment, participants had to watch 3D movies for a total of $30 \mathrm{~h}, 1-1.5 \mathrm{~h}$ per session, at least thrice a week, within six weeks in our research laboratory. Our treatment used a computer system capable of presenting 3D movies. A 24-inch computer screen (model, VG248QE; resolution, $1920 \times 1080$; refresh rate, $144 \mathrm{~Hz}$; ASUS, China) and a pair of active liquid crystal shutter glasses (model, cs-vs3 kit; Canshine, China) was used to display stereoscopic movies with a 3D player (Stereoscopic Player Version 1.6.4, 3dtv.at ), and parallax could be adjusted. The fellow eye was blurred with a Bangerter filter (Ryser Optik AG, Switzerland) to a level of roughly 0.2 logMAR worse than BCVA in the amblyopic eye, removing the obstacle to binocular vision while retaining the stereopsis at low and medium spatial frequencies.[33] The Bangerter filters used by the participants were based on our clinical measurements and adjusted as per the participant's vision changes. Children were encouraged to use the 1-m-long cable to ensure they maintained approximately $1 \mathrm{~m}$ viewing distance. They were allowed to view the screen at a shorter distance if they found it too blurry, but usually not less than $50 \mathrm{~cm}$.

\subsubsection{Patching Protocol}

The patching protocol was similar to the current standard of care for amblyopia treatment.[11] All participants received patching treatment, and amblyopia severity determined the dose of patching. Mild ( $<0.3 \log M A R)$, moderate (0.3 0.7 logMAR) and, severe ( $>0.7 \log M A R)$ amblyopia were prescribed 2,4 , and $6 \mathrm{~h}$ of patching per day, respectively. There was no difference between the treatment prescriptions of all patients and those before enrollment, but the actual patching time per day was subject to the parents' record.

\subsubsection{Adherence to Protocol}

The time and content of binocular treatment were recorded in electronic form. Legal guardians were provided a personalized calendar to record the minutes per day their child patched their fellow eye. 


\subsection{Statistical Analysis}

We anticipated a higher dropout rate for the combined treatment group; therefore, participants were allocated with a 3:2 ratio to the combined treatment group and the patching group, respectively. The primary outcome was a change in amblyopic eye BCVA at the 6-week visit. Based on previous studies, in the short term (6 weeks), the conventional patching resulted in a mean (SD) improvement of $0.10(0.10)$ $\log M A R[9,36]$, and the combined treatment resulted in a mean (SD) amblyopic-eye VA improvement of $0.20(0.10)$ logMAR.[34, 35] For $80 \%$ power with a type I error of 0.05 and $\leq 5 \%$ loss to follow-up, we planned to enroll 35 children ( 14 and 21 , respectively). All analysis included only participants completing the 6-weeks follow-up with an intent-to-treat approach. Stereo acuity (including Randot Preschool Test and Fly Stereo Acuity Test) was converted to log seconds of arc for analysis. The Randot Preschool Test and Worth 4 dot test results were combined into a binocular function score.[36] A value of 4.00 for nil stereopsis with fusion or partial suppression on the Worth 4 dot or 5.00 for nil stereopsis and suppression on the Worth 4 dot. The Worth 4-Dot test was conducted at a distance of 6 meters. Fly Stereo Acuity Test and Distance Stereopsis results were analyzed using a log-transformation with a value of 4.00 ( $\mathbb{Z}$ 400 arcsec) for nil stereopsis.

The primary outcome was amblyopic-eye BCVA improvement from baseline to 6 weeks. An independent ttest was conducted to determine whether improvement in amblyopic eye BCVA differed between the combined and the patching group. Repeated measures analysis of variance was conducted to examine whether amblyopic-eye BCVA of every group improved with time. A linear regression model was further used to analyze the associated factors to amblyopic-eye VA improvement. Wilcoxon test was conducted per group to determine whether stereo acuity had improved significantly from baseline at the 3 week and 6-weeks visits, respectively, for binocular function score, Fly Stereo Acuity Test, Distance Stereopsis results. Mann-Whitney test was conducted to determine the group differences. All tests were performed using a 2-tailed, $a=0.05$. 


\begin{tabular}{|c|c|c|c|}
\hline \multicolumn{4}{|c|}{$\begin{array}{c}\text { Table } 1 \\
\text { Baseline characteristics of included children among two groups }\end{array}$} \\
\hline & №. (\%). & & \\
\hline Characteristic & $\begin{array}{l}\text { Combined } \\
(n=18)\end{array}$ & $\begin{array}{l}\text { Patching } \\
(n=14)\end{array}$ & $\begin{array}{l}P \\
\text { value }\end{array}$ \\
\hline Female & $12(66)$ & $7(50)$ & 0.59 \\
\hline Age at enrolment & & & 0.12 \\
\hline Mean (SD), y & $6.90(1.66)$ & $6.65(0.93)$ & \\
\hline Range, y & $5.3-11.1$ & $5.1-8.9$ & \\
\hline Prior ptching treatment time & & & 0.17 \\
\hline Mean (SD), m & 19.11(16.31) & $12.71(5.37)$ & \\
\hline Type of amblyopia & & & 0.63 \\
\hline Anisometrope & $13(72)$ & $11(79)$ & \\
\hline Esotropia & $4(22)$ & $3(21)$ & \\
\hline Combined & $1(1)$ & $0(0)$ & \\
\hline Amblyopic-eye BCVA & & & 0.45 \\
\hline Mean (SD), logMAR & $0.48(0.16)$ & & \\
\hline Range, logMAR & $0.44(0.14)$ & & \\
\hline
\end{tabular}

y: year; m: month; BCVA: best-corrected visual acuity; logMAR: logarithm of the minimum angle of resolution.

\section{Results}

\subsection{Baseline characteristics}

A total of 35 participants were enrolled in this study between June 26, 2020, and October 29, 2021, and randomly assigned to the combined $(n=21)$ and patching groups $(n=14)$. The 6-weeks visit was completed by 18 participants (86\%) in the combined group and 14 participants $(100 \%)$ in the patching group (Figure 1). The clinical characteristics of the participants with amblyopia are shown in Table 1(See Supplementary material 1 for more detailed information), which were similar to baseline characteristics. Seven children (22\%) had strabismic amblyopia, 24 (75\%) had anisometropic amblyopia, and one (3\%) had combined mechanism amblyopia. The mean (SD) age was 6.79 (1.38) years (ranging between $5.1-11.1$ years), and 19 (59\%) were female. 


\subsection{Visit completion}

Three children completed baseline measurements but withdrew from the treatment study before three weeks. The reason given by the two children was an inconvenience to get to the laboratory, while the other child gave no explanation.

The researchers recorded compliance for Binocular treatment using an electronic form, and the patching adherence relied on the parental report. After six weeks, the mean total dose of binocular treatment was $32.4 \pm 4.9 \mathrm{~h}$ in the combined group. All patients in the combined group completed more than $80 \%$ of

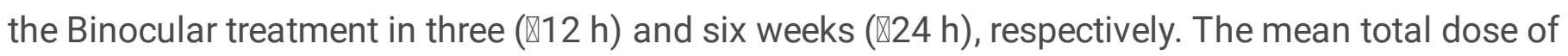
patching was $161 \pm 53 \mathrm{~h}$ in the combined group and $167 \pm 54 \mathrm{~h}$ in the patching group. Five (28\%) participants in the combined group and four $(29 \%)$ in the patching group completed less than the prescribed treatment. Combining the two groups of data, we found a significant negative correlation between age and patching time. $(r=-0.50, p=0.004)$.

\subsection{Acuity}

Figure $2 \mathrm{~A}$ and $2 \mathrm{~B}$ plots show the BCVA changes in the combined and patching groups, respectively, from the baseline to 3-week and 6-week visits. The individual values in the combined and patching groups measured before starting and after 6-week treatment (entry versus exit logMAR acuity) are plotted in Figures $2 C$ and $2 D$, respectively. The mean improvement in BCVA of $A E$ in each group compared with baseline at 3 and 6-week follow-up is shown in Figure 3A.

At the 6-week primary outcome visit, mean amblyopic-eye BCVA in the combined group improved from $0.49 \pm 0.16$ to $0.31 \pm 0.17 \log M A R$, with a significant mean gain of $0.17 \pm 0.08 \log M A R(2$-sided $95 \% \mathrm{Cl}$, 0.13 to $0.22 ; F=57.2, p \otimes 0.01)$. Amblyopic-eye BCVA in the patching group improved slightly with the mean gain of $0.05 \pm 0.04 \operatorname{logMAR}(2$-sided $95 \% \mathrm{Cl}, 0.05$ to $0.09 ; \mathrm{F}=8.73, p=0.01$ ). The value and improvement of amblyopic-eye BCVA of every group at different visit times are shown in Table 2 . The difference between two groups was statistically significant (mean difference, 0.13 logMAR [1.3 line]; 95\% $\mathrm{Cl}, 0.08-0.17$ logMAR [0.8-1.7 lines]; $\left.t_{25}=5.65, \mathrm{p}<0.01\right)$. For the combined group, eight children $(44 \%)$ improved by at least 0.2 logMAR ( 2 lines), and seven children (39\%) reached interocular acuity difference $($ IOAD ) $\leq 0.2$ logMAR (improved to normal levels). For the patching group, only two children (14\%) improved by at least $0.1 \log M A R$, and no child improved by more than $0.2 \log M A R$. Age and initial BCVA of AE were not significantly correlated $(r=0.01, p=0.99 ; r=0.11, p=0.66$, respectively) with the improvement of amblyopic-eye BCVA. (Figure 3B and 3C).

\subsection{Stereo acuity}

At 6 weeks, children in the combined group had significantly improved binocular function score (median [interquartile range], 2.30 [2.23 to 2.68] vs. 1.69 [1.60 to 2.30] log arcsec; paired, $z=-3.53, p \otimes 0.01$ ), and 
mean stereo acuity gain was $0.47 \mathrm{log} \operatorname{arcsec}( \pm 0.22)$. With the patching group (median [interquartile range], 2.30 [2.00 to 2.68] vs. 2.00 [1.78 to 2.68] log arcsec; paired, $z=-2.55, p=0.01$ ), mean stereo acuity gain was $0.14 \log$ arcsec $( \pm 0.13)$. The difference between the two groups was statistically significant $(z=-3.67, \mathrm{p} \otimes 0.01)$. Prior studies found the test-retest reliability of stereo acuity measurements using the Preschool Randot test in children to be $0.60 \mathrm{log}$ arcsec, with "real change" defined as doubling stereo acuity expressed in 1.99 octaves.[37] Eight children (44\%) in the combined group improved by at least $0.60 \mathrm{log}$ arcsec following treatment; the reason for the small improvement in four children was a better stereo vision at the baseline. Nine (50\%) patients reached a normal binocular function score (1.60 log arcsec, equivalent to 40 arcsec with Randot Preschool Stereoacuity Test). Binocular function scores changed in the combined group from baseline to 3-week, and 6-week visits are plotted in Figure 4A. Figure 4B shows the individual score in combined group entry versus exit log arcsec. Fly Stereo Acuity Test and Distance Stereo acuity value of children in the combined group also showed a significant improvement (see Table 2 for details). Only children with measurable stereo acuity at baseline showed improved stereo acuity. 
Table 2

Amblyopic-eye visual, stereoacuity in two treatment groups

$\begin{array}{lll}\text { Outcome } & \text { Combined } & \text { Patching } \\ & (n=18) & (n=14)\end{array}$

Amblyopic-eye BCVA, mean (SD), logMAR

Baseline

$0.49(0.16)$

$0.44(0.15)$

3-week visit

0.37(0.17)

0.43(0.14)

6-week visit

$0.31(0.17)$

$0.40(0.16)$

Amblyopic-eye BCVA improvement, mean (95\% Cl), logMAR

$\begin{array}{lll}\text { 3-week visit } & 0.12(0.09 \text { to } 0.15) & 0.01(-0.03 \text { to } 0.05) \\ \text { 6-week visit } & 0.17(0.13 \text { to } 0.22) & 0.05(0.00 \text { to } 0.10)\end{array}$

Interocular acuity difference, mean (SD), logMAR

$\begin{array}{lll}\text { Baseline } & 0.46(0.15) & 0.43(0.13) \\ \text { 3-week visit } & 0.35(0.16) & 0.42(0.13) \\ \text { 6-week visit } & 0.31(0.16) & 0.39(0.13)\end{array}$

Interocular acuity difference improvement, mean (95\% Cl), logMAR

3-week visit $\quad 0.12(0.08$ to 0.15$) \quad 0.01(-0.03$ to 0.05$)$

6-week visit $\quad 0.15(0.12$ to 0.19$) \quad 0.04(0.00$ to 0.08$)$

Stereoacuity

Binocular function score, median [interquartile range], log (seconds of arc)

Baseline $\quad 2.30(2.23$ to 2.68$) \quad 2.30(2.00$ to 2.68$)$

3-week visit $\quad 2.00(1.73$ to 2.60$) \quad 2.15(2.00$ to 2.68$)$

6-week visit $\quad 1.69(1.60$ to 2.30$) \quad 2.00(1.78$ to 2.68 )

Binocular function score improvement, mean (95\% Cl), log (seconds of arc)

3-week visit $\quad 0.29(0.18$ to 0.40$) \quad 0.06(-0.01$ to 0.12$)$

6-week visit $\quad 0.47(0.36$ to 0.59$) \quad 0.14(-0.06$ to 0.21$)$

FLYS stereoacuity, median [interquartile range], log (seconds of arc)

Baseline $\quad 2.30(2.15$ to 2.95$) \quad 2.30(2.00$ to 2.95$)$

3-week visit $\quad 1.90(1.67$ to 2.60$) \quad 2.25(2.00$ to 2.95$)$

6-week visit $\quad 1.60(1.30$ to 2.23$) \quad 2.10(1.80$ to 2.95$)$ 


\begin{tabular}{|c|c|c|}
\hline 3-week visit & $0.45(0.24$ to 0.66$)$ & $0.01(-0.01$ to 0.03$)$ \\
\hline 6-week visit & $0.71(0.48$ to 0.94$)$ & $0.12(0.06$ to 0.18$)$ \\
\hline \multicolumn{3}{|c|}{ Distance stereopsis, median [interquartile range], log (seconds of arc } \\
\hline Baseline & $2.29(2.13$ to 2.95$)$ & $2.28(1.95$ to 2.95$)$ \\
\hline 3-week visit & $1.90(1.70$ to 2.58$)$ & $2.23(1.94$ to 2.94$)$ \\
\hline 6-week visit & $1.70(1.30$ to 2.20$)$ & $2.13(1.78$ to 2.93$)$ \\
\hline \multicolumn{3}{|c|}{ Distance stereopsis improvement, mean ( $95 \% \mathrm{Cl}), \log$ (seconds of ar } \\
\hline 3-week visit & $0.43(0.23$ to 0.63$)$ & $0.03(0.01$ to 0.05$)$ \\
\hline 6-week visit & $0.74(0.51$ to 0.98$)$ & $0.15(0.07$ to 0.23$)$ \\
\hline
\end{tabular}

BCVA: best-corrected visual acuity; logMAR: logarithm of the minimum angle of resolution; FLYS: Fly Stereo Acuity Test.

\section{Discussion}

In this study, a set of equipment was provided that supports a three-dimensional (3D) display. Thus, amblyopia patients could watch their choice of movies in an immersive 3D environment containing larger disparities than generally encountered in natural scenes, combined with Bangerter blur filter to blur the fellow eye to rebalance the dominance of the two eyes. Previous studies reduced the suppression of the amblyopic eye by reducing the contrast of the input signal of the fellow eye. Here, we used the Bangerter blur filter to personalize the Gaussian blur level applied to the image viewed by FE during movie viewing. We observed substantial gains in acuity at our study and other studies[29, 38], indicating that fixed levels of blur and contrast penalization are both effective for treatment. The difference is that we used the Bangerter filter to get the BCVA of the fellow eye always 0.2 logMAR lower than the amblyopic eye, which may overcome the loss of monocular information in amblyopic eyes caused by fusion inhibition. When information from the two eyes is combined, and the binocular disparity is used to decode depth, monocular position information is lost, a phenomenon called fusional suppression.[39] In patients with amblyopia, fusional suppression is asymmetric. When patients viewed the vernier test target with the stronger of their two eyes, their vernier thresholds in the other eye were barely affected by the stereo halfimage. When they viewed the test target with their weaker eye, fusional suppression appears. In addition, in the contrast rebalance training used in previous studies, the treatment effect may be lost when it reaches $100 \%$. Our research design can prevent this from happening.

Although many studies have shown that binocular treatment can effectively improve patients' vision and other visual functions, two large randomized controlled trials have demonstrated inferior performance of binocular treatment relative to standard treatment (Patching).[35, 40] In addition, binocular therapy 
cannot be recommended to replace standard amblyopia therapy.[27] However, some patients showed poor response or compliance to traditional patching treatments in the clinic and often required higher doses and extended treatment periods. Age and compliance are related to treatment response.[41, 42] Therefore, it is necessary to include additional treatment methods to improve their vision and other visual functions to avoid missed effective treatment periods due to low compliance and responsiveness, resulting in permanent low vision. The patients included in our study all received traditional patching treatments before but failed to get a normal BCVA. However, this novel binocular treatment can improve the BCVA of the amblyopic eye and stereo acuity in a short period.

After six weeks of treatment, the mean increase in BCVA was 0.17 logMAR, and the difference was significant. Zhale[43] showed an improvement of $0.17 \log M A R$ in 1 month in children under combined patching with Interactive Binocular Treatment (I-BiT) for a total of $6.6 \mathrm{~h}, 20 \mathrm{~min}$ per session, five times a week. In addition, Yao[34] showed an improvement of 0.30 logMAR in 3 months in children under combined patching with the binocular game based on a push-pull method for $40 \mathrm{~min}$ a day (divided into two training sessions). Thus, we concluded that the combination of binocular treatment and patching could effectively improve the BCVA of children with amblyopia. Unfortunately, a dose-response relationship between binocular treatment and improvement of BCVA is still not reported.

Previous research on Dichoptic contrast balancing training, including I-BiT Dichoptic falling blocks game[44, 45] and Dichoptic iPad game (Dig Rush)[35, 40,46], had no convincing evidence of improvements in stereo acuity. They had a common feature: binocular treatment that balances the eyes by separating the eyes and reducing the contrast stimulation of the fellow eye without providing depth perception. Our research, in the form of 3D movies, provided rich visual stimulation and constantly changing parallax. The Randot Preschool Stereo acuity Test and the Fly Stereo Acuity Test were used for at near stereo acuity, and stereo acuity with was evaluated with 3D shutter glasses technology at distance. After treatment, the stereo acuity measured by the above three measurement methods had significantly improved. Our research results were consistent with Bossi et al.[38] which showed that six of seven children (with measurable stereo acuity at baseline) had significantly improved stereo acuity. Research by Bridgeman[28] and Li et al.[29] gave sufficient evidence suggesting that a 3D environment with large parallax may help improve stereo acuity in patients with amblyopia. However, our research showed that only patients with measurable stereo acuity at baseline had improved stereo acuity. In contrast, research by Bossi found that three stereo blind patients improved stereopsis after one year of binocular training. Therefore, to explore the potential therapeutic effect of our binocular treatment method on patients with stereoblindness, a longer follow-up study is needed. In a group where amblyopic eye visual acuity improvements are necessarily small, hard to achieve, and of questionable relevance, the restoration of binocular vision is a significant achievement. Restored binocular vision has fusion ability and the desired 3D ability, conducive to improving amblyopic children's fine motor skills. [36] It helps them feel the real world, as mentioned by Bridgeman in his personal experience.

There is no consensus on the best way to quantify interocular suppression; our study did not measure interocular suppression. Whether and how binocular therapy is related to the suppression of amblyopia is 
still very controversial. Some researchers believe that binocular treatment achieves the therapeutic effect by reducing interocular depression $[44,45]$ however, some studies have shown that interocular suppression is not reduced with the improvement of visual acuity and binocular vision in amblyopic eyes. $[38,47]$ We guess that during binocular treatment, the treatment of the contralateral eye (whether by reducing its contrast sensitivity or Gaussian blur) helps to reduce interocular suppression, but when this treatment is removed, the mitigation effect of interocular suppression will not last. It is no doubt that there is a great correlation between interocular suppression and amblyopia. Even though the therapeutic effect of amblyopia may not be related to the reduction of interocular suppression, it is likely to be related to the degree of initial interocular suppression. Earlier studies have shown that people with amblyopia with positive treatment have lower interocular inhibition than those with negative treatment.[48] More research is needed to explore the relationship between interocular suppression and the treatment of amblyopia and amblyopia.

Based on our laboratory research, the frequency and duration of binocular treatment are well documented. There is no significant difference between the children who received binocular treatment for $1.5 \mathrm{~h}$ each time, thrice a week (ID $3,6,8,10)$ and those who received the treatment for $1 \mathrm{~h}$ each time, five times a week (ID4, 11, 12,14). We also observed that the improvement in BCVA of AE in the first $15 \mathrm{~h}$ is greater than the last $15 \mathrm{~h}$. However, the sample size of this study is too small to carry out statistical analysis to prove the difference. Our research showed that there were significant differences in individual responses to binocular treatment. A patient (ID3) whose parents reported poor compliance with patching, after 15 and $30 \mathrm{~h}$ treatments, BCVA significantly improved to $0.26 \log M A R$ and $0.36 \log M A R$, respectively. Two children (ID7 and ID15) showed poor results after six weeks of treatment and received an additional $30 \mathrm{~h}$ of treatment; however, their BCVA of AE still did not improve. The timing of starting binocular therapy and its customized dosage is still a major clinical problem.

Furthermore, we can conclude that this binocular treatment is effective for part of patients with residual amblyopia. In children whose BCVA no longer improved after long-term traditional patching treatment, nothing is lost by trying this new type of short-term treatment. Moreover, there is no difficulty in realizing this treatment at home in the modern age. The high compliance of the combined group showed that this treatment is acceptable to both parents and children. In the future, the reasons for persistent amblyopia should be further explored in detail, and a larger sample size and longer follow-up study should be designed to explore the dose-response relationship of binocular treatment. We hope to develop a predictive model for the treatment response of amblyopia, refine the treatment frequency and each treatment dose, and look forward to individualized evaluation and treatment of patients with residual amblyopia in the clinic.

\section{Conclusions}

Our laboratory-based binocular treatment strategy engaged high levels of compliance and led to substantial gains in visual function after a short period of treatment. Especially it showed a greater advantage in improving the stereo acuity. At the same time, this treatment could become a means of 
family therapy. However, the specific therapeutic dose and duration of therapy still need to be studied further.

\section{Declarations}

\section{Author Contributions}

All authors contributed to the study conception and design. Qing Zhu participated in the design of the study, carried out the study and drafted the manuscript and performed the statistical analyses. Qi Zhao has participated in the study's coordination and has helped to draft the manuscript and has been involved in revising the manuscript carefully. Material preparation, data collection were performed by Ran Liang, Xing He and Mingjun Gao.

\section{Funding}

This study was funded by the Life Science Society of Liaoning.

\section{Data availability}

The data used to support the findings of this study are included within the article and supplementary material .

\section{Conflict of interest}

The authors declare that they no conflict of interest.

\section{Ethical approval}

All procedures performed in studies involving human participants were in accordance with the ethical standards of the second hospital of Dalian Medical University and with the 1964 Helsinki Declaration and its later amendments or comparable ethical standards.

\section{Consent to participate}

Informed consents were obtained from the subjects after explanation of the nature of the study.

\section{Consent to publish}


The parents of patients agreed to collect and publish the data of their children.

\section{References}

1. M. Y. Frenkel and M. F. Bear, "How monocular deprivation shifts ocular dominance in visual cortex of young mice," Neuron, vol. 44I, no. 6l, pp. 917-923I, 2004.

2. K. Iny, et al., "Bidirectional modifications of visual acuity induced by monocular deprivation in juvenile and adult rats," The Journal of neuroscience : the official journal of the Society for Neuroscience, vol. 26I, no. 28I, pp. 7368-7374l, 2006.

3. D. Giaschi, et al., "Sparing of coarse stereopsis in stereodeficient children with a history of amblyopia," Journal of vision, vol. 13l, no. 10l, pp. 2013.

4. A. R. O'Connor, et al., "Relationship between binocular vision, visual acuity, and fine motor skills," Optometry and vision science : official publication of the American Academy of Optometry, vol. 87l, no. 12l, pp. 942-947I, 2010.

5. C. Huang, et al., "Treated amblyopes remain deficient in spatial vision: a contrast sensitivity and external noise study," Vision research, vol. 47I, no. 11, pp. 22-34l, 2007.

6. M. X. Repka, et al., "A randomized trial of patching regimens for treatment of moderate amblyopia in children," Archives of ophthalmology (Chicago, III. : 1960), vol. 121I, no. 5I, pp. 603-611l, 2003.

7. J. M. Holmes, et al., "A randomized trial of prescribed patching regimens for treatment of severe amblyopia in children," Ophthalmology, vol. 110l, no. 111, pp. 2075-2087l, 2003.

8. "A randomized trial of atropine vs. patching for treatment of moderate amblyopia in children," Archives of ophthalmology (Chicago, III. : 1960), vol. 120l, no. 3l, pp. 268-278I, 2002.

9. R. P. Rutstein, et al., "A randomized trial comparing Bangerter filters and patching for the treatment of moderate amblyopia in children," Ophthalmology, vol. 117l, no. 5l, pp. 2010.

10. M. X. Repka, et al., "Two-year follow-up of a 6-month randomized trial of atropine vs patching for treatment of moderate amblyopia in children," Archives of ophthalmology (Chicago, III. : 1960), vol. 123I, no. 2l, pp. 149-157l, 2005.

11. C. E. Stewart, et al., "Treatment dose-response in amblyopia therapy: the Monitored Occlusion Treatment of Amblyopia Study (MOTAS)," Investigative ophthalmology \& visual science, vol. 45I, no. 9l, pp. 3048-3054l, 2004.

12. G. Woodruff, et al., "Factors affecting the outcome of children treated for amblyopia," Eye (London, England), vol. 8 ( Pt 6)l, no. pp. 627-6311, 1994.

13. E. M. Rislove, et al., "Scale-dependent loss of global form perception in strabismic amblyopia," Journal of vision, vol. 10I, no. 12I, pp. 25I, 2010.

14. J. M. Holmes, et al., "Risk of amblyopia recurrence after cessation of treatment," Journal of AAPOS : the official publication of the American Association for Pediatric Ophthalmology and Strabismus, vol. 8I, no. 5I, pp. 420-428I, 2004. 
15. R. Bhola, et al., "Recurrence of amblyopia after occlusion therapy," Ophthalmology, vol. 113l, no. 11l, pp. 2097-2100l, 2006.

16. R. Farivar, et al., "Interocular suppression in strabismic amblyopia results in an attenuated and delayed hemodynamic response function in early visual cortex," Journal of vision, vol. 11l, no. 14l, pp. 2011.

17. E. E. Birch, "Amblyopia and binocular vision," Progress in retinal and eye research, vol. 33l, no. pp. 6784l, 2013.

18. Y. El-Shamayleh, et al., "Visual motion processing by neurons in area MT of macaque monkeys with experimental amblyopia," The Journal of neuroscience : the official journal of the Society for Neuroscience, vol. 30l, no. 36l, pp. 12198-12209l, 2010.

19. L. Kiorpes, et al., "Neuronal correlates of amblyopia in the visual cortex of macaque monkeys with experimental strabismus and anisometropia," The Journal of neuroscience : the official journal of the Society for Neuroscience, vol. 18I, no. 16I, pp. 6411-6424I, 1998.

20. O. Joly and E. Frankó, "Neuroimaging of amblyopia and binocular vision: a review," Frontiers in integrative neuroscience, vol. 8l, no. pp. 62I, 2014.

21. R. F. Hess, et al., "Binocular vision in amblyopia: structure, suppression and plasticity," Ophthalmic \& physiological optics : the journal of the British College of Ophthalmic Opticians (Optometrists), vol. 34l, no. 2l, pp. 146-162I, 2014.

22. A. T. Astle, et al., "The pattern of learned visual improvements in adult amblyopia," Investigative ophthalmology \& visual science, vol. 52I, no. 10l, pp. 7195-7204l, 2011.

23. J. Ding and D. M. Levi, "Recovery of stereopsis through perceptual learning in human adults with abnormal binocular vision," Proceedings of the National Academy of Sciences of the United States of America, vol. 108I, no. 37I, pp. E733-E7411, 2011.

24. D. M. Levi, et al., "Stereopsis and amblyopia: A mini-review," Vision research, vol. 114l, no. pp. 17-30l, 2015.

25. R. F. Hess, et al., "A new binocular approach to the treatment of amblyopia in adults well beyond the critical period of visual development," Restorative neurology and neuroscience, vol. 28I, no. 6l, pp. 793-802l, 2010.

26. I. Tsirlin, et al., "Behavioral Training as New Treatment for Adult Amblyopia: A Meta-Analysis and Systematic Review," Investigative ophthalmology \& visual science, vol. 56I, no. 6l, pp. 4061-4075I, 2015.

27. S. L. Pineles, et al., "Binocular Treatment of Amblyopia: A Report by the American Academy of Ophthalmology," Ophthalmology, vol. 127l, no. 2I, pp. 261-272I, 2020.

28. B. Bridgeman, "Restoring adult stereopsis: a vision researcher's personal experience," Optometry and vision science : official publication of the American Academy of Optometry, vol. 911, no. 6l, pp. e135e139l, 2014.

29. R. W. Li, et al., "Improving Adult Amblyopic Vision with Stereoscopic 3-Dimensional Video Games," Ophthalmology, vol. 125I, no. 10l, pp. 1660-1662I, 2018. 
30. K. Zhao and X. Shi, "[Learn new version of Preferred Practice Pattern to further standardize the diagnosis and treatment of amblyopia]," [Zhonghua yan ke za zhi] Chinese journal of ophthalmology, vol. 50l, no. 7l, pp. 481-484I, 2014.

31. X.-Y. Liu, et al., "Dichoptic Perceptual Training in Children With Amblyopia With or Without Patching History," Investigative ophthalmology \& visual science, vol. 62I, no. 6l, pp. 4I, 2021.

32. H. Wu, et al., "Evaluating stereoacuity with 3D shutter glasses technology," BMC ophthalmology, vol. 16I, no. 1l, pp. 45I, 2016.

33. R. W. Li, et al., "Monocular blur alters the tuning characteristics of stereopsis for spatial frequency and size," Royal Society open science, vol. 3l, no. 9l, pp. 160273I, 2016.

34. J. Yao, et al., "Binocular game versus part-time patching for treatment of anisometropic amblyopia in Chinese children: a randomised clinical trial," The British journal of ophthalmology, vol. 104l, no. 3l, pp. 369-375l, 2020.

35. J. M. Holmes, et al., "Effect of a Binocular iPad Game vs Part-time Patching in Children Aged 5 to 12 Years With Amblyopia: A Randomized Clinical Trial," JAMA ophthalmology, vol. 134I, no. 12I, pp. 1391-1400l, 2016.

36. A. L. Webber, et al., "Fine Motor Skills of Children With Amblyopia Improve Following Binocular Treatment," Investigative ophthalmology \& visual science, vol. 57I, no. 11I, pp. 4713-4720I, 2016.

37. W. E. Adams, et al., "Defining real change in measures of stereoacuity," Ophthalmology, vol. 116l, no. 2l, pp. 281-285I, 2009.

38. M. Bossi, et al., "Binocular Therapy for Childhood Amblyopia Improves Vision Without Breaking Interocular Suppression," Investigative ophthalmology \& visual science, vol. 58I, no. 7l, pp. 30313043l, 2017.

39. S. P. McKee and R. A. Harrad, "Fusional suppression in normal and stereoanomalous observers," Vision research, vol. 33I, no. 12l, pp. 1645-1658l, 1993.

40. V. M. Manh, et al., "A Randomized Trial of a Binocular iPad Game Versus Part-Time Patching in Children Aged 13 to 16 Years With Amblyopia," American journal of ophthalmology, vol. 186l, no. pp. 104-115l, 2018.

41. J. M. Holmes, et al., "Effect of age on response to amblyopia treatment in children," Archives of ophthalmology (Chicago, III. : 1960), vol. 129I, no. 11I, pp. 1451-1457I, 2011.

42. S. E. Loudon, et al., "Electronically measured compliance with occlusion therapy for amblyopia is related to visual acuity increase," Graefe's archive for clinical and experimental ophthalmology = Albrecht von Graefes Archiv fur klinische und experimentelle Ophthalmologie, vol. 241I, no. 3l, pp. 176-180l, 2003.

43. Z. Rajavi, et al., "The role of Interactive Binocular Treatment system in amblyopia therapy," Journal of current ophthalmology, vol. 28I, no. 4I, pp. 217-222I, 2016.

44. S. L. Li, et al., "A binocular iPad treatment for amblyopic children," Eye (London, England), vol. 281 , no. 10l, pp. 1246-1253l, 2014. 
45. E. E. Birch, et al., "Binocular iPad treatment for amblyopia in preschool children," Journal of AAPOS : the official publication of the American Association for Pediatric Ophthalmology and Strabismus, vol. 19l, no. 1l, pp. 2015.

46. T. Y. Gao, et al., "Effectiveness of a Binocular Video Game vs Placebo Video Game for Improving Visual Functions in Older Children, Teenagers, and Adults With Amblyopia: A Randomized Clinical Trial," JAMA ophthalmology, vol. 136l, no. 2l, pp. 172-181I, 2018.

47. K. R. Kelly, et al., "Binocular iPad Game vs Patching for Treatment of Amblyopia in Children: A Randomized Clinical Trial," JAMA ophthalmology, vol. 134l, no. 12I, pp. 1402-1408I, 2016.

48. S. Narasimhan, et al., "Quantitative measurement of interocular suppression in children with amblyopia," Vision research, vol. 66I, no. pp. 2012.

\section{Figures}

\section{Figure 1}

General Study Design. Shown are the numbers of completed and missed visits during the 6-week study. Combined group: patching treatment combined binocular therapy; Patching group: patching treatment alone.y: year; n: number; Aniso:anisometropic amblyopia; Strab: strabismus amblyopia(Including strabismus combined with anisometropic )

\section{Figure 2}

$(a, b) B C V A$ in the AE during treatment in the combined and patching groups. Participants' amblyopia type was pure anisometropic ([a]: $n=13 ;[b]: n=11$, circles), pure strabismic, or combined ([a]: $n=5 ;[b]: n=3$, triangle). A color represents each individual, age indicated in parentheses, in legends of parts $[a, b]$. Identity codes ([a]:1-18; [b]: 1-14) are given next to individual lines and in the legends of (a) and (b) and label individual data points in (c) and (d). (c, d) Comparison of pre-and post-treatment acuity for each child in the combined and patching groups, respectively. Points below the diagonal line are an improvement, with the shaded region indicating gains less than $0.15 \log M A R$ (considered critical of testretest reliability).

\section{Figure 3}

(a) The mean improvement in BCVA of AE in each group compared with baseline at 3 and 6-weeks followup. $(b, c)$ Age and initial BCVA of AE were not significantly correlated $(r=0.01, p=0.99 ; r=0.11, p=0.66$, 
respectively) with the improvement of amblyopic-eye BCVA.

\section{Figure 4}

(a) Stereo acuity (binocular function score) in the combined group for children with measurable stereopsis at baseline who completed treatment. Before, during, and after treatment (at 0, 3, and 6 weeks, respectively). (b) Pre-versus post-treatment. For eight participants, stereo acuity gains exceeded the testretest variability threshold of $0.6 \log$ arcsec and a step of 1.99 octaves (shaded area).

\section{Supplementary Files}

This is a list of supplementary files associated with this preprint. Click to download.

- BaselineDetailsofParticipants.xIsx 